\title{
Cement Debonding: Is it a Problem with Antibiotic Cement Coated Nails?
}

\author{
Somasekhara Reddy Nallamilli, MS, MCh ${ }^{{ }^{*}}$, Rajyalakshmi N Reddy, MS ${ }^{2}$ and Mohan Krishna Althuri ${ }^{1}$ \\ ${ }^{1}$ Consultant Orthopaedic Surgeon, International pavilion, Apollo Hospital, India \\ ${ }^{2}$ Registrar in Orthopaedics, Apollo Hospital, India
}

\begin{abstract}
It is a common practice to use antibiotic impregnated cement coated intramedullary nails, Rush pins and other metal rods to treat infection in the medullary canal which may be secondary to open injuries or following previous internal fixations or prosthetic replacement surgeries. They helped to control the infection and also to act as interim fixators and prosthetic spacers. There are several reports of debonding or delamination of the cement from the underlying metal implant. Not much was written about the problems the debonded cement can create and difficulties in removing it which often is a pre requisite for the subsequent definitive procedure. The problem is worse with retained bits of cement if infection persisted. Some precautions were advised to avoid the same while preparing and inserting the antibiotic cement coated nails but there are no special implants designed to prevent de-bonding. We describe a simple technique to improve the hold of the cement on to the underlying metal so that debonding won't happen.
\end{abstract}

\section{Keywords}

Antibiotic cement nails, Non-articulating antibiotic cement hip prosthesis, Open fracture with antibiotic cement coated nails (ACN), Cement debonding, Cement delamination, Retained cement in the bone medullary canal

\section{Introduction}

Acute or sub-acute infection of a medullary canal is well known following intramedullary nail fixations of long bone fractures. Infection may be more common after open fractures and it may persist even after fracture union. The infection may settle down after removal of the metal implant but in some cases, it may not. Some cases may even develop chronic osteomyelitis which may not respond to oral or parenteral antibiotics. Similar infection may involve hip joint and proximal femur following total hip replacement and hemiarthroplasty of the hip joints. Paley, et al. popularised a technique by preparing an intramedullary nail coated with antibiotic-impregnated cement [1]. Several techniques were subsequently described to prepare these antibiotic cement nails [2-6]. However, these implants share a common problem of cement debonding where large pieces of cement mantle may come off the nail while inserting or more commonly they are left behind in the medullary canals when these metal rods are explanted. These bits may interfere with subsequent definitive management of fracture fixation or replacement. Even though some precautions were described to minimise the risk of de-bonding, there is no special method or special implants which can prevent cement de-bonding in these cases. We describe a technique which has helped in preventing cement de-bonding in our cases.

\section{Material and Methods}

Five patients were included in this study where the improvised antibiotic-impregnated cement coated nails (ACN) were used (Table 1). Two patients presented with infection after total hip replacement surgery, one patient after hemiarthroplasty. Two patients presented with infection following type II open fractures of both bones of the leg which were earlier treated with external fixators elsewhere and both cases had adequate soft tissue cover when they presented to us. These two patients refused to continue with external fixators and requested for alternate ways of management. Informed consent was taken about the likely duration of treatment, chances of the persistence of infection and multiple procedures. At first stage of surgery, hip implants were explanted, thorough debridement was carried out and custom made antibiotic ce-

*Corresponding author: Somasekhara Reddy Nallamilli, MS, MCh, Consultant Orthopaedic Surgeon, International pavilion, Apollo Hospital, \#957, Jubilee Hills, Hyderabad - 500033, Telangana, India, Tel: +919848025355

Accepted: September 09, 2020

Published online: September 11, 2020

Citation: Nallamilli SR, Reddy RN, Althuri MK (2020) Cement Debonding: Is it a Problem with Antibiotic Cement Coated Nails?. J Orthop Surg Tech 3(2):143-149 
ment coated implant was inserted. For infected tibial fracture cases, medullary canal was reamed and after thorough wash, ACNs were inserted; adequate stability of the fractures was obtained with removable below-knee splints following surgery. Patients were mobilised non-weight bearing. Patients received antibiotics for a total period of 6 weeks. Serial measurements of ESR and CRP were carried out until 8 weeks. In all the five cases these values remained in the normal range after cessation of antibiotic treatment and clinically they did not show any signs of active infection. Then they were taken

Table 1: Details of the cases in this study, all of which were referred from other centres.

\begin{tabular}{|c|c|c|c|c|c|}
\hline Sex & $\begin{array}{l}\text { Age } \\
\text { (Y) }\end{array}$ & Primary condition & Organism & $\begin{array}{l}\text { Antibiotic added to bone } \\
\text { cement (DePuy CMW } 1 \\
\text { Gentamicin cement) }\end{array}$ & $\begin{array}{l}\text { Post Op antibiotic treatment } \\
\text { for } 6 \text { weeks }\end{array}$ \\
\hline Male & 49 & THR Infected & Staph. aureus & $\begin{array}{l}\text { Vancomycin } 2 \mathrm{gm} \text { per } 40 \mathrm{gm} \\
\text { cement }\end{array}$ & Inj Co-amoxyclav $1.2 \mathrm{gm}$ bd \\
\hline Female & 34 & THR Infected & Staph. aureus & $\begin{array}{l}\text { Vancomycin } 2 \mathrm{gm} \text { per } 40 \mathrm{gm} \\
\text { cement }\end{array}$ & Inj Co-amoxyclav $1.2 \mathrm{gm}$ bd \\
\hline Female & 54 & $\begin{array}{l}\text { Hemiarthroplasty of Hip } \\
\text { joint, Infected }\end{array}$ & K. pneumoniae & $\begin{array}{l}\text { Vancomycin } 2 \mathrm{gm} \text { per } 40 \mathrm{gm} \\
\text { cement }\end{array}$ & $\begin{array}{l}\text { Inj Cefoperazone }+ \\
\text { Sulbactam1.5 gm bd }\end{array}$ \\
\hline Male & 27 & $\begin{array}{l}\text { Type II open fracture both } \\
\text { bones of leg }\end{array}$ & Staph. epidermidis & $\begin{array}{l}\text { Vancomycin } 2 \mathrm{gm} \text { per } 40 \mathrm{gm} \\
\text { cement }\end{array}$ & Inj Co-amoxyclav $1.2 \mathrm{gm}$ bd \\
\hline Male & 29 & $\begin{array}{l}\text { Type II open fracture both } \\
\text { bones of leg }\end{array}$ & Enterococcus sp & $\begin{array}{l}\text { Vancomycin } 2 \mathrm{gm} \text { per } 40 \mathrm{gm} \\
\text { cement }\end{array}$ & $\begin{array}{l}\text { Inj Cefoperazone + Sulbactam } \\
1.5 \mathrm{gm} \text { bd }\end{array}$ \\
\hline
\end{tabular}

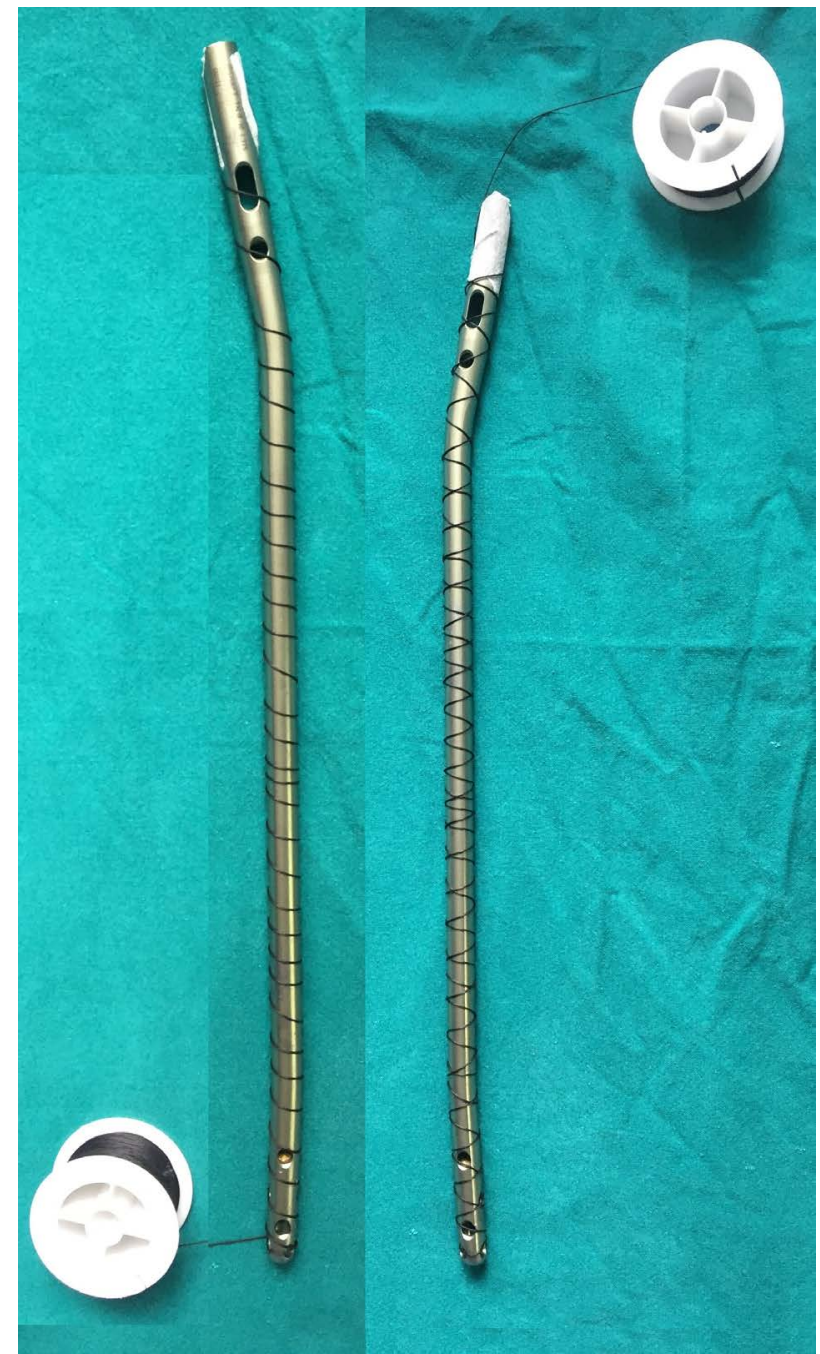

Figure 1: Preparation of a nail with no $22 \mathrm{G}$ wire wound around it from one end to the other end and then in reverse direction.

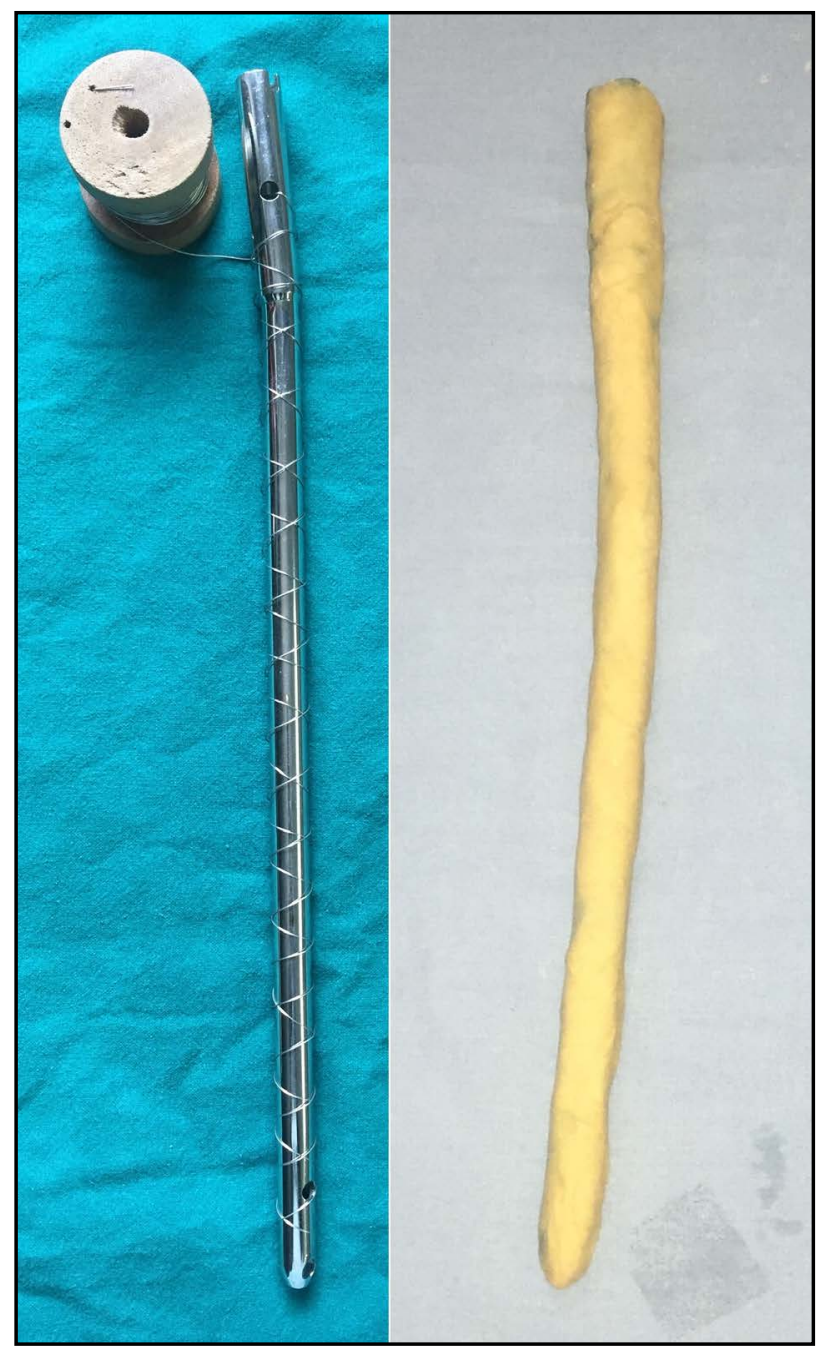

Figure 2: Preparation of antibiotic impregnated cement coated nail. 
up for second stage surgery. In all cases, the ACNs were explanted fully intact. Re-debridement, medullary canal reaming and wash out was carried out before proceeding for definitive procedure. Two infected hip cases (one with previous THR and one with the previous hemiarthroplasty) had revision total hip replacement. One case of previously infected THR case got lost for follow up and turned for second stage revision THR after 3 years. Tibial fracture cases had interlocking intramedullary nailing.

\section{Selection of bone cement and preparation}

Culture and sensitivity reports of fluid from the infected hip joints and swabs form the sinuses over the hip and legs were considered for selection of appropriate antibiotics after discussing with the bacteriologist. We used DePuy CMW 1 (40 gm with gentamicin $1 \mathrm{gm}$ ) cement. For the cases included in this study, we added $2 \mathrm{gm}$ of Vancomycin for 40 gm pack of cement.

\section{The technique of preparation of antibiotic ce- ment coated nail}

Preparation involved two steps. First, select the appropriate nail-length and diameter and prepare with cerclage wire. Second, apply antibiotic mixed bone cement to the desired purpose.

\section{Intramedullary interlocking nail}

Select a thinnest possible nail. The ideal diameter is at least $4 \mathrm{~mm}$ less than the last reamer used. No. 20 or No. 22

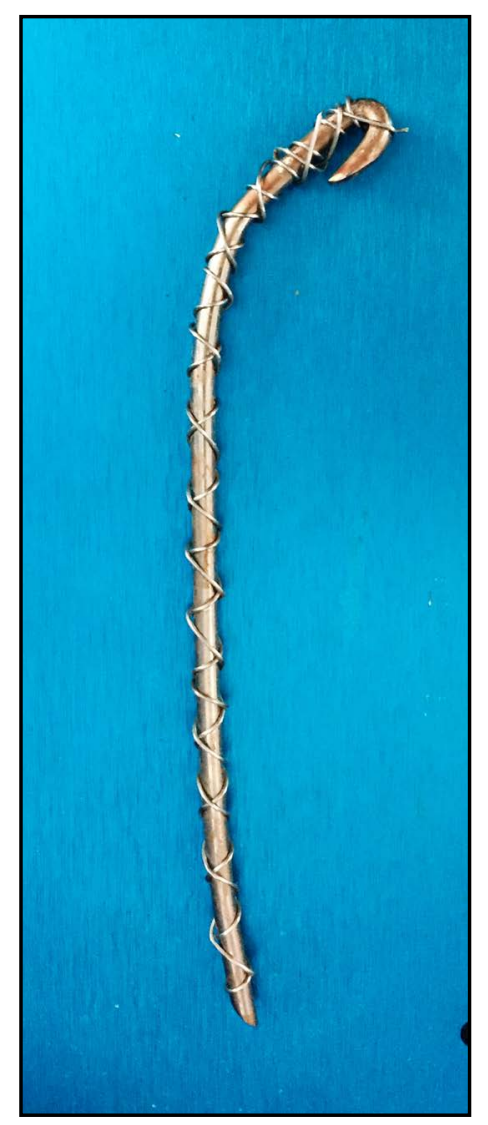

Figure 3: A bent Rush nail with No $18 \mathrm{G}$ wire wound around it to prepare a hemiarthroplasty $\mathrm{ACN}$.
G stainless steel cerclage wire is selected. Stabilize the tip of the cerclage wire at one end of the nail with a sterile adhesive tape and wind it around the nail till the other end of the nail. Once the cerclage wire reaches the distal end of the nail, then wind it back to the proximal end of the nail. Then cut the cerclage wire and twist the ends of the wire together after removing the adhesive tape (Figure 1). If the medullary canal is unusually narrow then a $6 \mathrm{~mm}$ Rush nail is selected and no. $22 \mathrm{G}$ wire was used for winding. Cement was hand-rolled on the prepared nail/Ender's nail and it was passed through a preselected diameter hole of a metal gauze. The forward and backward movement through the corresponding diameter of the gauze was done several times while the cement is setting to prepare ACN of uniform diameter (Figure 2).

\section{Non-articulating proximal femur prosthesis}

For cases of infected hemi-arthroplasty or total hip joint replacement, a Rush nail is bent to about 120 degrees at neck-shaft angle and No. 18 or No. 20 G stainless steel wire was used to wind around it as described above (Figure 3 ). Then antibiotic mixed cement was applied on to the prepared nail. The shape and diameter of the medullary canal of the proximal femur and bone defects in trochanter are carefully assessed and the implant is prepared to occupy maximum space within the canal but nearly conical to be able to extract easily. Once or twice a trial insertion was done into the proximal femur while the cement is still soft to make sure it goes-in and come-out easily (Figure 4).

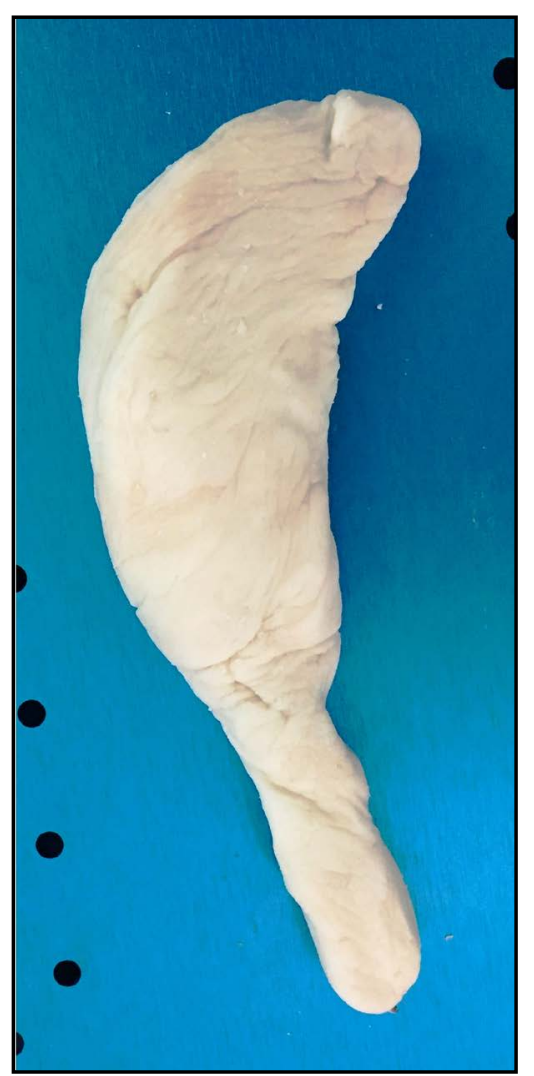

Figure 4: Antibiotic impregnated cement coated Rush nail for proximal femur implant. 


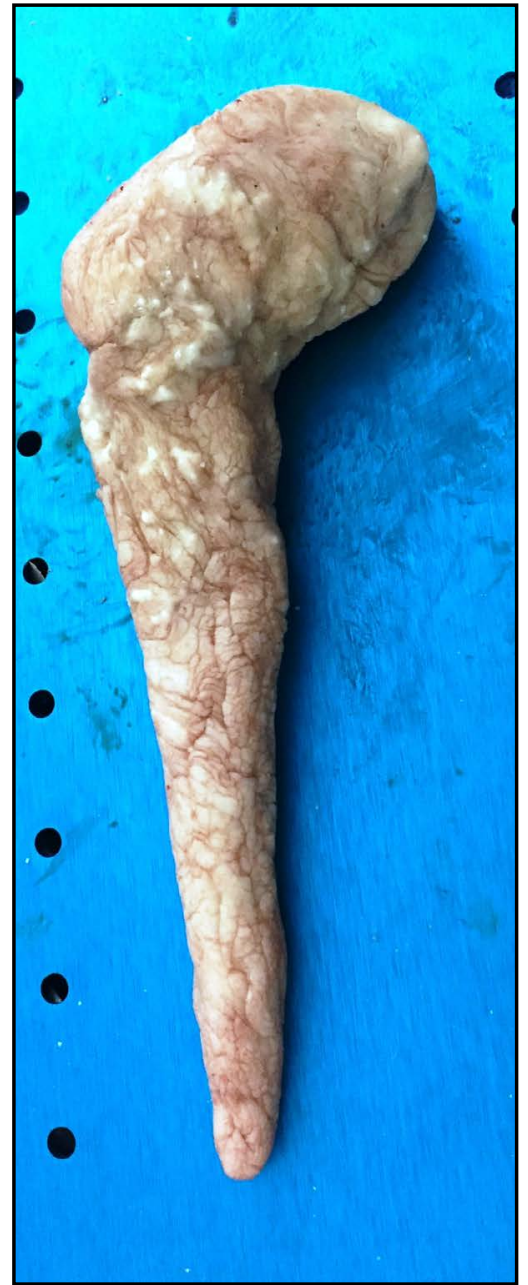

Figure 5: A non-articulating upper femur ACN prosthesis, explanted fully intact.

\section{Results}

We tried the improvised technique of preparation of antibiotic cement coated nails for three non-articulating hip prostheses and two tibia nails which were referred to us from other centres. Preparation of ACNs using smooth nails is slightly difficult as the cement slides over the smooth nail rather than sticking to it where it was applied. The nails we made with cerclage wires provided anchorage to the cement making it comparatively easy to prepare the ACN. We have not seen debonding of cement mantle while preparing the nail, inserting the nail or while removing it (Figure 5). In one case the patient did not come back for definitive surgery for three years after the first stage (Figure 6). Removal of the cement stem from the femoral medullary canal was difficult due to fibrous in-growth around the cement. A plane was created around the ACN with flexible osteotomes in the proximal femur and the cement implant came out fully intact despite forced rotations and telescoping manoeuvres to pull it out. All the five cases had control of infection. Tissue samples taken at second stage did not grew any organisms and had successful results with satisfactory hip revisions and healed tibia fractures. Results of this article were limited to the issues related to the cement debonding.

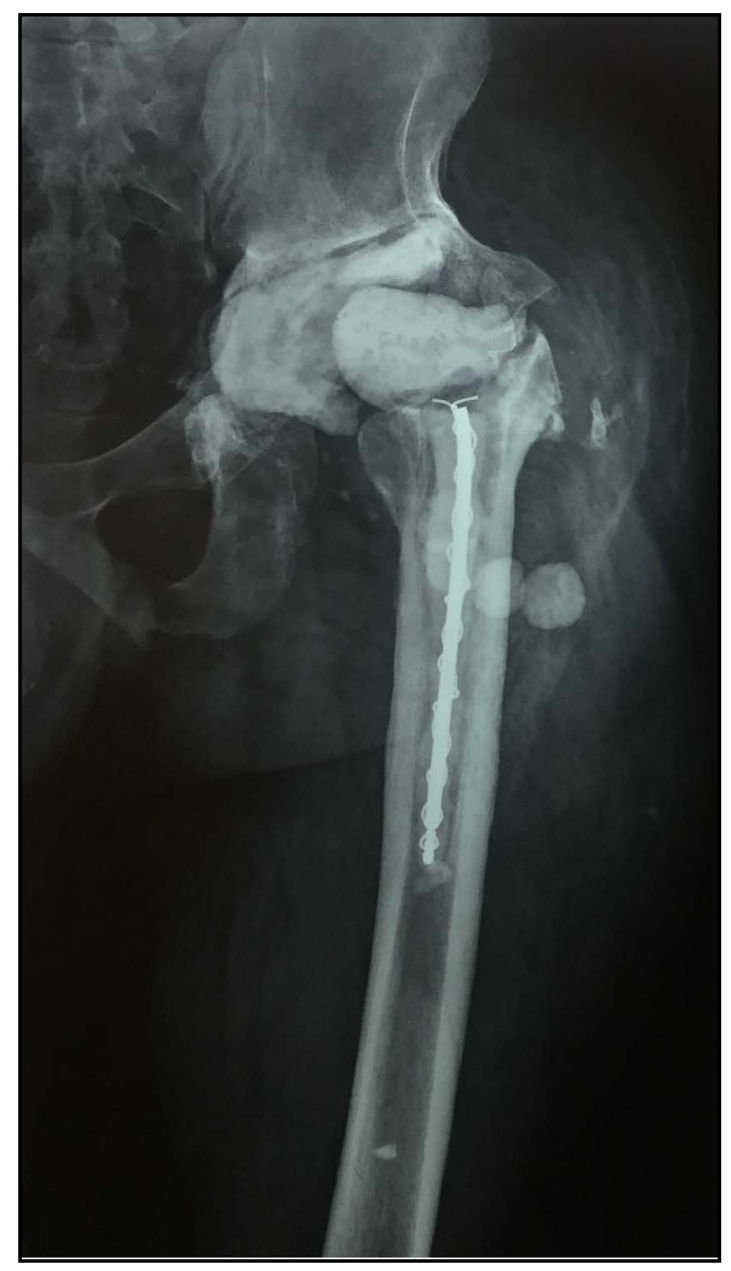

Figure 6: Radiograph of ACN in upper femur three years after insertion.

\section{Discussion}

Cases with intramedullary locking nail fixations with or without a history of an open fracture and cases of either a total or hemiarthroplasty of the hip joint may present with infection. The treatment invariably starts with extensive debridement of the soft tissues in the hip joint and reaming of medullary canals of femur or tibia. Antibiotic impregnated cement spacers were successfully introduced to treat infected joint replacement surgeries [7]. The same theory was extended to treat the intramedullary infection of long bones as antibiotic-impregnated cement coated nails (ACN) can take high concentrations of antibiotic to the full length of the medullary canal and offers some degree of immediate stability which in itself can help control the infection [1]. Wasko, et al. and Makridis, et al. wrote extensively on various aspects of ACNs and to avoid repetition, the same was avoided here and readers are referred to those studies for full information. But an important problem with ACNs is not addressed in the literature though many papers reported the complication of cement de-bonding of ACNs $[6,8]$.

Kuntscher nails are used commonly for femur $[2,5,6]$. Chavan, et al. suggested that the slot of K-nail accommodates a good amount of cement and resists easy de-bonding; Debond- 


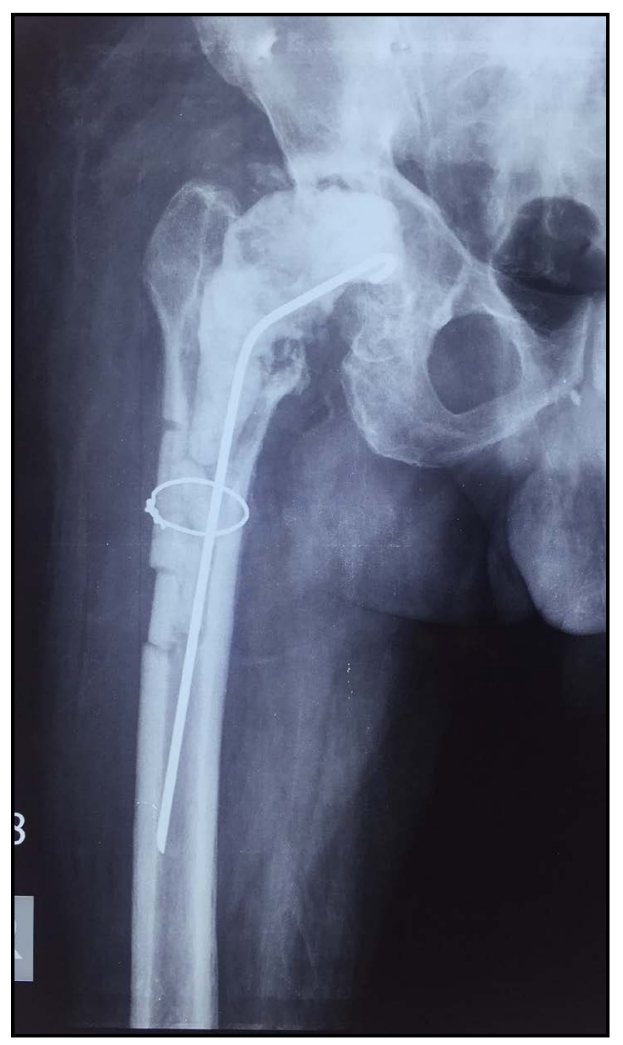

Figure 7: Cement de-bonding from a smooth Rush nail while inserting with almost no cement in the lower half of the nail (Courtesy Dr. Aachi).

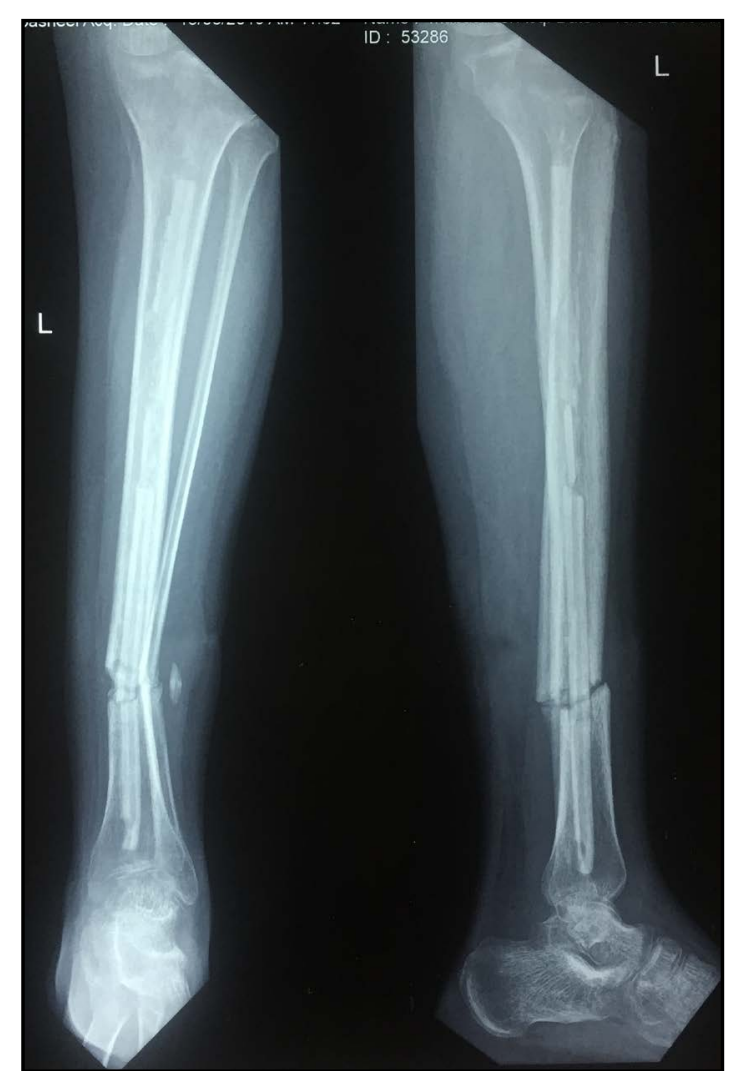

Figure 8: A tibial non-union with retained cement mantle through the entire medullary canal.

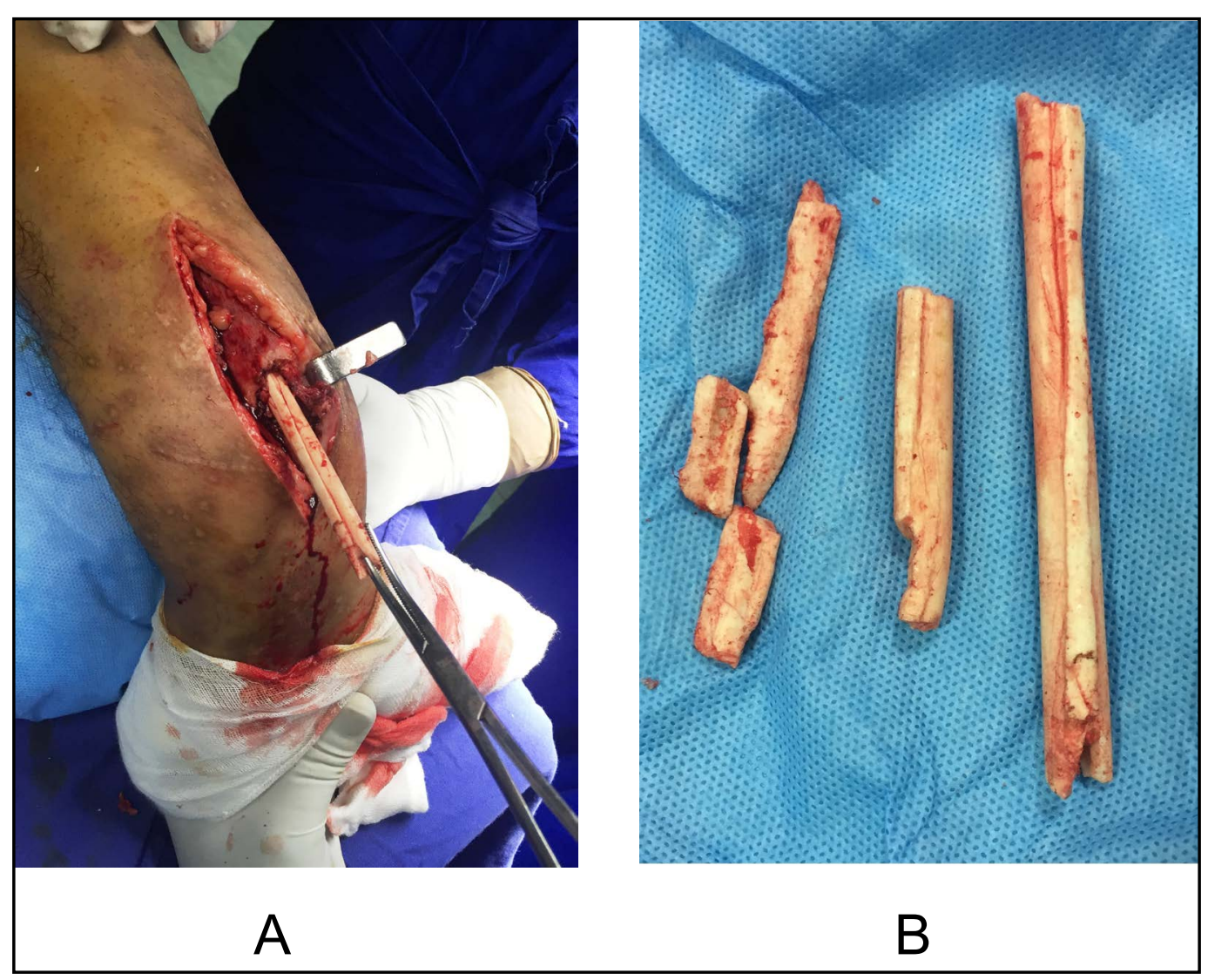

Figure 9: (a) Removal of the pieces of cement mantle after opening the fracture; (b) A picture of the removed cement mantle tubes. 
ing is common with canulated non-slotted nails, though matt finished nails may offer some advantage [2]. Some authors recommended allowing time for the cement to set and bond well with the nail, adequate over reaming, to drive-in the nail with hand and not to hit with a hammer while inserting the nail to avoid cement debonding $[4,5,9]$. Once the infection is under control the cement coated implant needs to be removed and a definitive fixation is done. However, cement debonding occurs to varying degrees despite all the above precautions. There can be de-bonding of the cement from the metal implant while preparing, inserting-commonly in cases with segmental defects (Figure 7), Courtesy Dr. Aachi, or more commonly identified at the time of its removal [3]. Thonse, et al. reported debonding in $20 \%$ of cases with two cases of retained cement in the medullary canal [3]. Saravanan, et al. reported 2 cases of de-bonding out of 25 cases [9]. There are other studies reporting cement de-bonding in the literature, though details of numbers were not given. Hence they were not included in this article. Our hospital being a tertiary care centre sometimes we get cases with retained pieces of cement in the bones after their initial treatment with ACNs elsewhere. In one case the primary surgeons treated an open fracture of both bone of the leg with an ACN in the tibia. They successfully controlled the infection and got the soft tissue cover. At planned second stage surgery the smooth Ender's nail which was used for preparing the ACN slipped out of the tibia leaving the entire cement mantle inside the medullary canal (Figure 8). He was then referred to our centre. This poses a difficult problem which is under-reported in the literature. If the fracture has healed well and the infection is fully under control perhaps the remaining pieces of cement in the medullary canal will be of no significance. However, if the infection still lingered on, the procedure may need to be re-done with another ACN. For that, the medullary canal needs to be thoroughly cleared of any residual cement which in itself can be a source of infection. Many times the infection may have cleared but the fracture may still have not united and it may need definitive fixation. Unless the pieces of cement mantle left in the canal are not removed, no further definitive procedure can be done. Attempts to remove the bits of cement using revision arthroplasty cement removal instruments may be successful in some cases. In cases where the fracture has not united, exposing the fracture site may serve a dual purpose of open removal of the intramedullary cement and for freshening and bone grafting of the fracture site (Figure 9a and Figure 9b) along with definitive fixation of the fracture, which we did for the above case.

To prevent these complications we devised the technique described above. Though the presented series is small, we are convinced with its mechanical advantage and have helped our colleagues to prepare such nails for their infected cases. Kenneth, et al. used a nail with a threaded core [10]. Woo Cho, et al. used threaded rods in 40 cases and reported no cement debonding or delamination in their cases and easier implant removal [11]. The core principle in techniques of ACN preparation in both the reports is to bond cement to the nail in a more effective way by using a threaded rod. We achieved the same objective with our novel technique of cerclage wire around the nail and we had no case of cement de-bonding.
This technique is easy and involves no extra-inventory and no additional cost. It is also not easy to procure threaded rods. The true magnitude of cement de-bonding will be known only if surgeons identify and report such cases. A comparative study between preparation of ACNs with smooth and cerclage wire encircled nails in a large trauma centre will help confirm the effectiveness of the latter.

\section{Highlights}

- Cement debonding or delamination is a common problem while using antibiotic impregnated cement coated nails (ACN).

- Well recognised problem but under reported and no easy solutions are forthcoming.

- Potential problems include persistence of infection as the residual cement in the medullary canal may act as a source of infection.

- Subsequent definitive procedures cannot be done with the pieces of cement blocking the medullary canal.

- Simple modification of the present technique of preparing ACN will help to almost fully avoid the problem of cement de-bonding.

- Since it is a small series, authors suggest a large study from a dedicated trauma unit to confirm the usefulness of this novel, inexpensive technique.

\section{Acknowledgement}

Mr. Yedukondalu (late), a senior technician for his skills in preparing the ACNs and training surgeons and other technicians.

\section{References}

1. Paley D, Herzenberg JE (2002) Intramedullary infections treated with antibiotic cement rods: Preliminary results in nine cases. J Orthop Trauma 16: 723-729.

2. Chavan V, Bairwa VK, Jhanwar P, et al. (2019) Role of antibiotic-impregnated cement intramedullary nail in infected nonunion of long bone diaphyseal fractures. J Orthop Traumatol Rehabil 11: 16-20.

3. Thonse R, Conway J (2007) Antibiotic cement-coated interlocking nail for the treatment of infected non-unions and segmental bone defects. J Orthop Trauma 21: 258-268.

4. Shyam AK, Sancheti PK, Patel SK, et al. (2009) Use of antibiotic cement-impregnated intramedullary nail in treatment of infected non-union of long bones. Indian J Orthop 43: 396-402.

5. Bhatia C, Tiwari AK, Sharma SB, et al. (2017) Role of antibiotic cement coated nailing in infected nonunion of tibia. Malays Orthop J 11: 6-11.

6. MK Wasko, O Borens (2013) Antibiotic cement nail for the treatment of posttraumatic intramedullary infections of the tibia: Midterm results in 10 cases. Injury 44: 1057-1060.

7. Wilde AH, Ruth JT (1988) Two-stage reimplantation in infected total knee arthroplasty. Clin Orthop Relat Res 236: 23-35.

8. K Makridis, T Tosounidis, P Giannoudis (2013) Suppl 2: Management of infection after intramedullary nailing of long bone frac- 
tures: Treatment protocols and outcomes. The Open Orthopaedics Journal 7: 219-226.

9. Saravanan A, R Raj Ganesh, N Deen Muhammed Ismail, et al. (2017) Antibiotic cement impregnated nailing in management of infected non-union of femur and tibia. International Journal of Scientific Study 5: 187-191.
10. Kenneth L Koury, John S Hwang, Michael Sirkin (2017) The antibiotic nail in the treatment of long bone infection: Technique and results. Orthop Clin North Am 48: 155-165.

11. Jae Woo Cho, Jinil Kim, Won Tae Cho, et al. (2018) Antibiotic coated hinged threaded rods in the treatment of infected nonunions and intramedullary long bone infections. Injury 49: 19121921. 